\section{EMBRYRIDDLE Aeronautical University}

SCHOLARLY COMMONS

\section{International Journal of Aviation,} Aeronautics, and Aerospace

\title{
Support to Design for Air Traffic Management: An Approach with Agent-Based Modelling and Evolutionary Search
}

\author{
Gabriella Gigante \\ CIRA (Italian Aerospace Research Centre), g.gigante@cira.it \\ Roberto Palumbo \\ CIRA (Italian Aerospace Research Centre), r.palumbo@cira.it \\ Domenico Pascarella \\ CIRA (Italian Aerospace Research Centre), d.pascarella@cira.it \\ Alessandro Pellegrini \\ Sapienza University of Rome, pellegrini@diag.uniroma1.it \\ Gabriella Duca \\ ISSNOVA (Institute for Sustainable Society and Innovation), duca@issnova.eu \\ Miquel Angel Piera \\ Universitat Autònoma De Barcelona, miquelangel.piera@uab.cat \\ Juan José Ramos \\ Universitat Autònoma De Barcelona, juanjose.ramos@uab.es
}

Follow this and additional works at: https://commons.erau.edu/ijaaa

Part of the Computational Engineering Commons, Management and Operations Commons, and the Multi-Vehicle Systems and Air Traffic Control Commons

\section{Scholarly Commons Citation}

Gigante, G., Palumbo, R., Pascarella, D., Pellegrini, A., Duca, G., Piera, M., \& Ramos, J. (2021). Support to Design for Air Traffic Management: An Approach with Agent-Based Modelling and Evolutionary Search. International Journal of Aviation, Aeronautics, and Aerospace, 8(1). https://doi.org/10.15394/ ijaaa.2021.1561

This Article is brought to you for free and open access by the Journals at Scholarly Commons. It has been accepted for inclusion in International Journal of Aviation, Aeronautics, and Aerospace by an authorized administrator of Scholarly Commons. For more information, please contact commons@erau.edu. 


\section{Support to Design for Air Traffic Management: An Approach with Agent-Based Modelling and Evolutionary Search}

\section{Cover Page Footnote}

This work has received funding from the SESAR Joint Undertaking with grant agreement No 783189

(EvoATM project) under European Union's Horizon 2020 research and innovation program. 
To enhance Air Traffic Management (ATM) and meet the future traffic demand and environmental requirements, present ATM system is going to be modified (SESAR Joint Undertaking, 2017), designing new services to be integrated in future architecture considering the evolution of present fragmented structure of the airspace and the entanglement of air routes. Such a change process is complicated due to the nature of ATM, which is a large-scale Socio-Technical System (STS), typically involving a complex interaction between humans, machines and the environment. In such kind of systems, managing their evolution is a complex and difficult task since the social and technical implications of any proposed concept should be fully assessed before a choice is made whether or not to proceed with the related development. Often, simulation tools are also used to support the design of the concept itself by enabling what-if-analyses. However, these may be too effort and time consuming due to the exponential growth of the required analysis cases. A quite common mismatch between the performance evaluations in simulated conditions and those achieved in real life is represented by the partial assessment of human aspects that can be performed throughout the new concept lifecycle from its lowest maturity level up to "ready to market."

The proposed work defines an approach to support the design of new ATM solutions, including the evaluation on human behaviour. The approach adopts a combined paradigm, which involves Agent-Based Modelling and Simulation (ABMS) to specify and analyse the ATM models, and Agent-based Evolutionary Search (AES) to optimize the design of the new solutions. A specific case study is used to demonstrate the effectiveness of the proposed approach. Transition from Direct Routing Airspace (DRA) to Free Routing Airspace (FRA), respectively described by Solution \#32 and Solution \#33 in the SESAR solutions catalogue (SESAR Joint Undertaking, 2017), is used for both validation and experimentation activities. In detail, the proposed experimentation case regards the design of sector collapsing/decollapsing configuration to optimize controller workloads. The achieved results are presented and discussed.

\section{Literature Review}

Modelling socio-technical systems has been largely studied in the last decade. Different and interconnected trends are noteworthy to mention, such as system dynamics, Bayesian belief networks, ABMS, etc. In the last 10 years, ABMS (Macal, 2016) has been used for modelling complex systems in different domains, including ATM. The analysis of open literature shows multiple examples to solve ATM problems. Many commercial tools are available, e.g., SIMMOD (www.airporttools.com), AirTOp (www.airtopsoft.com), or the AgentFly ATM simulation (Šišlák et al., 2011). In the USA it is notable to mention the effort of NASA and FAA to develop FACET (Bilimoria et al., 2001), a simulation tool to assess air traffic management concepts. Most of the tools provide detailed models 
of airports and airspace for fast-time gate-to-gate simulation; very few use multiagent architectures for different actors of the scene, e.g. for airport controllers. None embeds the human behaviour modelling.

ABMS has been used to address a wide number of different ATM problems in several research projects within the SESAR Exploratory Research framework, such as ELSA (Bongiorno et al., 2015),ACCESS (Herranz, et al., 2015), TREE (Ciruelos et al., 2015), CASSIOPEIA (Molina et al., 2014), MAREA (Stroeve et al., 2013), SPAD (Pasquini et al., 2013), EMERGIA (Blom \& Bakker, 2016), and SAFECORAM (Palumbo et al., 2015). In these projects, ABMS is used (usually in combination with other techniques) either as an analysis tool to understand emergent behaviours of ATM systems (as in CASSIOPEIA, ELSA, and EMERGIA), or to study resilience and disturbance propagation (as in MAREA, SPAD, TREE, and SAFECORAM), or as a tool to determine airport slot auctioning and allocation (as in ACCESS). However, most of these works include only the modelling of technical aspects, not considering the social aspects that influence the overall performance of the ATM system.

Regarding AES (Sarker \& Ray, 2010), it is an emerging research paradigm lying at the intersection between evolutionary computing and ABMS, two paradigms that pledge to contribute together to the analysis and to the solution of challenging and complex problems in areas that span from online trading to disaster response. Depending on the research area, the combination of evolutionary computing and ABMS is still relatively unexplored, especially considering the study of socio-complex systems, in which the interaction between individual/cognitive, social, technical and environmental factors, generates complexities that are difficult to predict and be dealt with (Gilbert \& Troitzsch, 2005). In recent years, the combination of evolutionary computing and ABMS is gaining attention as a viable and innovative way to investigate complex adaptive systems (Yliniemi et al., 2014).

\section{Approach}

This work proposes an approach to answer the research problems concerning the automated support to design of a new ATM concept in order to achieve the required performance levels. We consider a scenario-based approach, where a scenario is intended as a description of the reference operating environment, including: a set of actors; a set of available actions; a set of processes; the relationships between the previous elements and their formalization as a flow of information, representing the dynamics to allow the system to perform a mission or a service. In detail, the proposed approach refers to a changed scenario, or solution scenario, or solution. This represents the scenario integrating the change for the ATM system of interest. 
As a case study, we considered the transition from Direct Routing Airspace (DRA) operations (reference scenario), to Free Routing Airspace (FRA) operations (changed scenario). DRA and FRA solutions are already specified in the SESAR solutions (SESAR Joint Undertaking, 2017) and are respectively described by solution \#32 ("free route through the use of direct routing") and solution \#33 ("free routing for flights both in cruise and vertically-evolving above a specified flight level in low-to-medium density airspace"). The selection of this case study is supported by the fact that the transition from DRA to FRA has already been tackled in the Italian airspace from 2013 to 2016, and therefore its known change and design history represents a meaningful resource for both validation and experimentation activities related to this work. This case study assumes the involvement of a limited set of actors which are modelled as agents in our approach. Specifically, the scenarios involve: Air Traffic (ATCOs), i.e., Executive Controllers (ECs) and Planner Controllers (PCs) across multiple sectors; Controller Working Positions (CWPs); aircraft; and Flight Crews (FCs).

Modelling specifications are structured using the FRAM (Functional Resonance Analysis Method) notation (Hollnagel, 2012). Such formalism provides an easy link between the ATM experts and the modelers, allowing a straightforward modelling for agent-based simulations. The workflow implemented for the support to design is shown in 
International Journal of Aviation, Aeronautics, and Aerospace, Vol. 8 [2021], Iss. 1, Art. 9

Figure 1. The first activity has been the FRAM-based specification of the solution scenario for the new concept (Step 1). Then, the solution concept has been analysed focusing on its design in a performance-based setting, which provides the statement of the design problem (Step 2). 


\section{Figure 1}

Proposed Workflow for the Support to Design

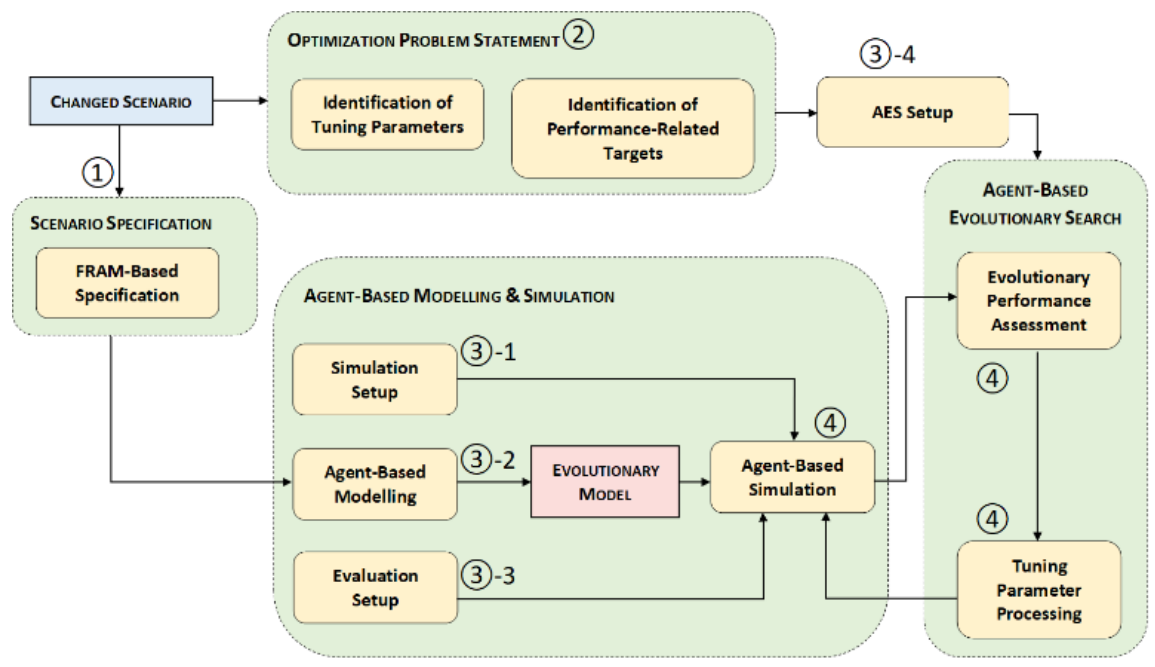

In the problem statement, target objectives have been identified in terms of expected values for the performance metrics and design attributes have been selected as numerical parameters, whose values have to be tuned to attain the target objectives. This sets up a constrained and multi-objective optimization problem, where the objectives are represented by the metrics and their target values, whereas possible constraints are represented by limitations on safety, airspace capacity or on other problem-related variables. The decision variables of the problem coincide with the selected tuning parameters.

The FRAM-based specification of the solution concept has been coded in the agent-based model of the solution (Step 3-2). This is also called evolutionary model since it is not fixed, but it is subjected to AES for the optimal tuning of design parameters. In parallel, the architecture for the agent-based simulation (Step 3-1) and the metrics evaluation module (Step 3-3) have been set up. Also, the part of evolutionary search has been arranged and the optimization problem has been properly coded (Step 3-4) by implementing the AES engine. Then, the AES engine has exercised the evolutionary model for the iterative optimization phase (Step 4).

The proposed approach exploits FRAM notation to specify a model for the sociality of agents, i.e., the information that each agent needs to exchange with the others in order to perform its assigned functions. In detail, our approach customizes the standard FRAM notation to adapt it as a "fastener" between the views of ATM experts (e.g., a specification by means of hierarchical task analysis) and agentbased modelling experts. With reference to Figure 2 (up), each FRAM component (i.e., the hexagon) represents an action of an agent. Each agent is specified as a course of FRAM actions, that is a structured sequence of agent's actions, whose 
execution impacts on the generic performance metrics $\mathrm{W}$ and $\mathrm{F}$ of the agent itself. The flow interactions among actions are expressed by means of FRAM interfaces as appropriate: for example, the output of an action may provide the input of another action, or may encode its resources, pre-conditions, or time horizon, etc. The social interactions between different agents are specified in the same way, i.e., they are connections between FRAM actions belonging to the course of distinct agents. Hence, scenarios are specified by customized FRAM components and by their interfaces considering the relationships between humans (i.e., human agents), technology (i.e., technical agents) and their environment. This ensures the coexistence of technical and social characteristics in the specification.

Regarding human agents, we have focused on modelling the human behaviour of the ATCO agents of the scenarios, specifically in their two roles of EC and PC. Conversely, FCs' behaviours have been modelled with a higher level of abstraction, using a characterization of the interactions between the FC and the controllers as specified by the standard procedures of the airline to which the FC belongs. Following a scenario-based approach, it has been possible to breakdown the structure of the ATCOs' tasks relative to the case study. Then, a Hierarchical Task Analysis (HTA) has provided an operational description of the selected scenario and the identification of tools, external conditions, triggers and outputs per each task, as well as the identification of relevant human behaviour variables and attributes. Figure 2 (down) shows an overview of the workflow and the final identified attributes and variables that characterize the controller agents. Most of these variables do not possess a strictly quantitative representation. However, to allow the simulation of the performance variability of human agents in different conditions, each variable is represented using discrete values, each value being a possible state of the personal, cognitive, and socio-cultural factors. 
Figure 2

Customization of FRAM Components for the Approach (up) and Human Performance Model for ATCOs (down)

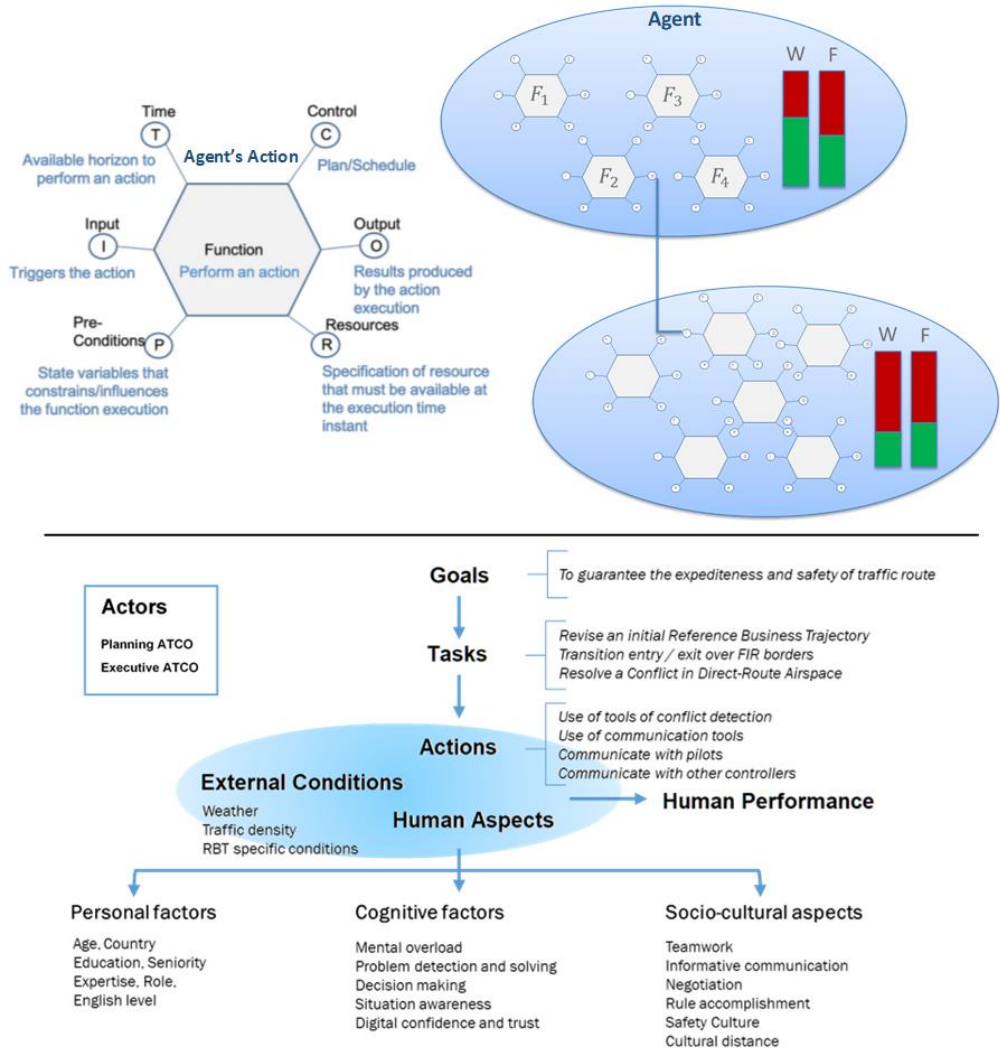

\section{Agent-Based Evolutionary Search}

The proposed approach exploits computational intelligence techniques, which apply AES to support the design of ATM solutions through the optimization of reference performance metrics. It addresses an evolutionary optimization architecture to carry out an optimization loop in order to continually modify the design parameters, which are expressed as input parameters of the simulation model. These modifications aim at the identification of properly tuned configurations to increase the overall expected performance of the ATM solution.

The optimization approach employs AES to explore the complex search space of the agent-based models, wherein architectural and design choices influence the ATM system at both macro and micro scales. Our optimization process is based on Evolutionary Algorithms (EAs), which are inspired by concepts from nature (e.g., evolution and natural selection) and provide effective heuristics for computationally intensive problems. They maintain a population of individuals 
(potential solutions), which compete for survival. New offspring are created by recombining and mutating individuals selected from the population.

The exploration of the overall search space is driven by multiple objective functions, which are related to the reference performance metrics and may include also variables associated to the human behaviour and controller workload. These functions address a multi-objective optimization problem whose solutions identify the best-suited configurations of the input parameters. The multiple objectives must be jointly optimized at the same time, so that the fitness function used to evaluate the goodness of the individuals is directly derived from these objectives. Thus, the requirements for the optimization approach are the following:

- $\quad$ There are multiple input variables to optimize and there are multiple output metrics to assess the goodness of a configuration. Thus, Multi-Objective EAs (MOEAs) is a proper choice for the design of the optimization framework.

- Optimization is simulation-based and the optimization architecture must consider non-minimal execution times. This requires a parallel/distributed and simulation-based optimization architecture.

In detail, our evolutionary computing solution is based on a parallel/distributed variant of NSGA-II (Non-dominated Sorting Genetic Algorithm II) algorithm (Deb et al., 2002). NSGA-II is a popular and widely used MOEA, which exhibits several properties that are fundamental to meet the requirements of optimization which we have set. The customization of NSGA-II has led to a parallel/distributed simulation-based architecture, which aims at a resolution of the optimization problem in a reduced amount of time. This architecture is based on the Master-Slave paradigm. The building blocks of the architecture (Figure 2) are the following:

- $\quad$ Orchestrator - It is the master and it distributes the amount of work across the available processors.

- Compute nodes - They are the nodes of the parallel/distributed infrastructure and they evaluate (through stochastic simulation) the goodness (fitness) of a tuple describing an individual of the population.

Compute metrics - This is a logical block which is executed on the compute node after that a batch of simulation is completed. This module computes the values of the performance metrics of interest.

- $\quad$ Selection and evolution - This tags all the individuals with the metrics and applies the evolutionary strategy to evolve the population towards the optimal configuration.

A more detailed description of this evolutionary computing algorithm and architecture is available in (Pellegrini, et al., 2020). 
Figure 2

Optimization Architecture for AES

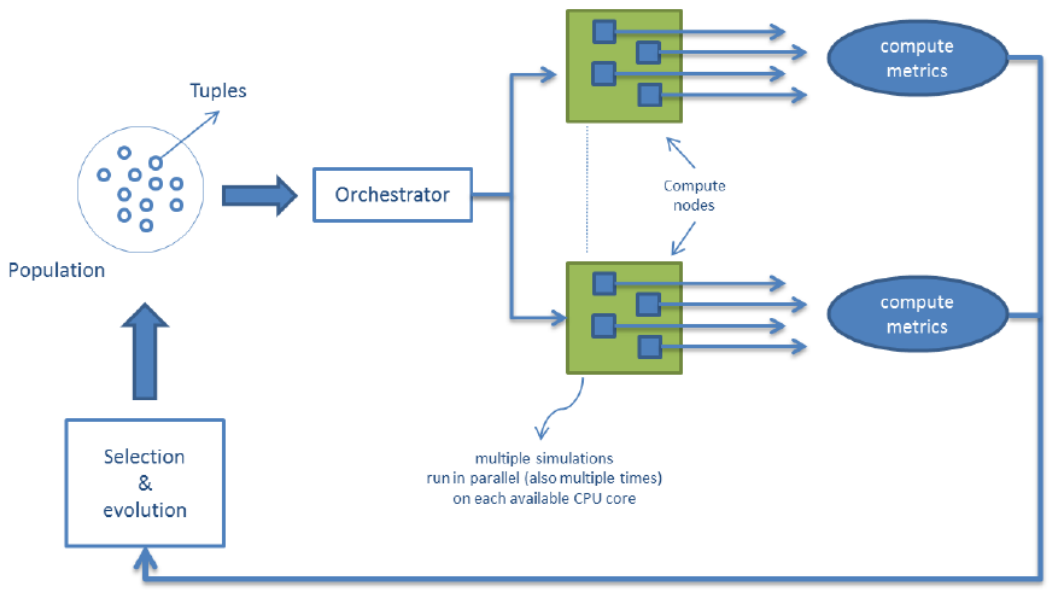

Validation of the Agent-Based Simulation Environment

This section gives an overview of the validation of the ABMS engine. Broadly speaking, validation is carried out selecting a known air traffic scenario, and then computing and comparing the simulated performance metrics with the performance metrics determined using real data. The known reference scenario is related to DRA operations over the Italian airspace. We considered flight operations during several days of 2016 in two different seasons (summer and winter). We also considered two different types of traffic complexities that involve different groups of airspace sectors. Specifically:

- $\quad$ a High Complexity (HC) scenario, that includes six collapsed sectors (LIBBND34, LIBBND57, LIBBCE34, LIBBCE57, LIBBES34, LIBBES57) of Brindisi ACC (LIBB), and which mainly captures the sliding traffic of LIBB, but also parts of the departing and landing traffic of the main southern Italian airports;

- $\quad$ a Very High Complexity (VHC) scenario, that includes six collapsed sectors (LIPPN34, LIPPN57, LIPPCS34, LIPPCS57, LIPPSD34, LIPPSD57) of Padua ACC (LIPP) and four collapsed sectors (LIMMWC34, LIMMWC57, LIMME34, LIMME57) of Milan ACC (LIMM), and which captures very complex and dense traffic patterns (sliding, climbing and descending) of LIMM and LIPP.

The ABMS engine simulates air traffic in these sectors starting from planned flight data. Results are then compared with actual flown data. Both planned and actual data are obtained from Demand Data Repository (DDR2) of EUROCONTROL. Validation is performed by analysing the punctual and the 
sampling/statistical differences between the performance metrics. The following trajectory-related metrics are considered:

- $\quad$ sector occupancy, evaluated as the number of flights in a sector within a timeframe of 10 minutes;

- $\quad$ sector flight timeliness, intended as permanence time of a flight in a sector;

in a sector.

These metrics can be easily determined also for real flight data. In contrast, human-related metrics (e.g., controller workloads), even if assessed for each simulation run, cannot be used for the comparison with real world performance because of lack of quantitative data. In any case, the chosen metrics reflect the need to capture the expected benefits of DRA and FRA solution. In fact, an analysis of the key performance areas impacted by DRA and FRA operations allowed to identify relevant key performance indicators and quantitative metrics. Figure 4 shows the relationships and interdependencies between the metrics, the key performance indicators and the key performance areas.

Figure 4

Mapping Between Key Performance Areas and Quantitative Metrics

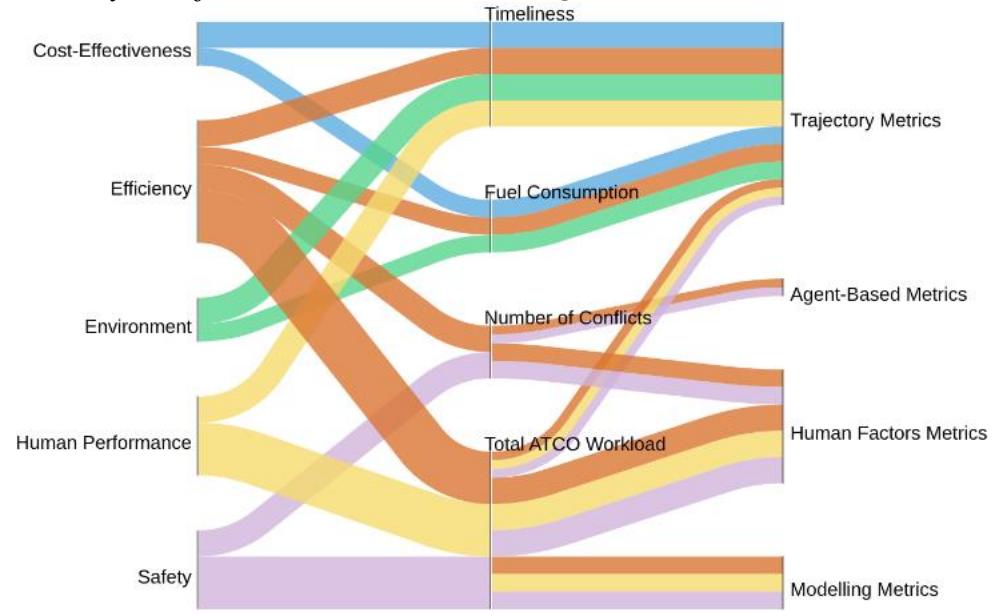

For the sake of brevity, Table 1 reports only the maximum errors measured for each day in each of the analysed traffic scenarios (over 8900 flights were considered). Validation results indicate good agreement between simulated and real flights, the maximum errors being small. Furthermore, expert controllers of the Italian ANSP (ENAV, Ente Nazionale per l'Assistenza al Volo) have been engaged for a discussion on the achieved results. The magnitude order of the metric 
assessment has been evaluated as likely and plausible by Italian experts, proving its acceptability from a validation perspective.

Moreover, this validation campaign exhibits a proper statistical coverage. Some examples are shown in Figure 5 to report the statistical characterizations of the errors for the assessment of the sector timeliness and the sector flight length within HC and VHC traffic of 06/07/2016.

\section{Table 1}

Maximum Errors Measured for Each Day (for both HC and VHC scenarios)

\begin{tabular}{|c|c|c|c|c|c|c|}
\hline $\begin{array}{c}\text { Overall Maximum } \\
\begin{array}{c}\text { Error } \\
\text { across Sectors }\end{array}\end{array}$ & \multicolumn{2}{|c|}{$\begin{array}{c}\text { Max Occupancy Error } \\
\text { [flights / 10 min] }\end{array}$} & \multicolumn{2}{|c|}{$\begin{array}{c}\text { Max Timeliness } \\
\text { Error } \\
\text { [min] }\end{array}$} & \multicolumn{2}{c|}{$\begin{array}{c}\text { Max Flight } \\
\text { Length Error } \\
\text { [NM] }\end{array}$} \\
\hline Scenario & $\boldsymbol{H C}$ & $\boldsymbol{V H C}$ & $\boldsymbol{H C}$ & $\boldsymbol{V H C}$ & $\boldsymbol{H C}$ & $\boldsymbol{V H C}$ \\
\hline 06 Jul 2016 & $<0.3$ & $<0.5$ & $<4$ & $<4$ & $<20$ & $<22$ \\
\hline 07 Jul 2016 & $<0.1$ & $<0.6$ & $<3$ & $<4$ & $<20$ & $<22$ \\
\hline 08 Feb 2016 & $<0.1$ & $<0.3$ & $<3$ & $<4$ & $<18$ & $<22$ \\
\hline 09 Feb 2016 & $<0.2$ & $<0.6$ & $<4$ & $<4$ & $<20$ & $<24$ \\
\hline
\end{tabular}

\section{Figure 5}

Confidence Interval (with a confidence level of 95\%) of the Errors in the Estimations of Sector Timeliness and Sector Flight Length for HC and VHC Scenarios

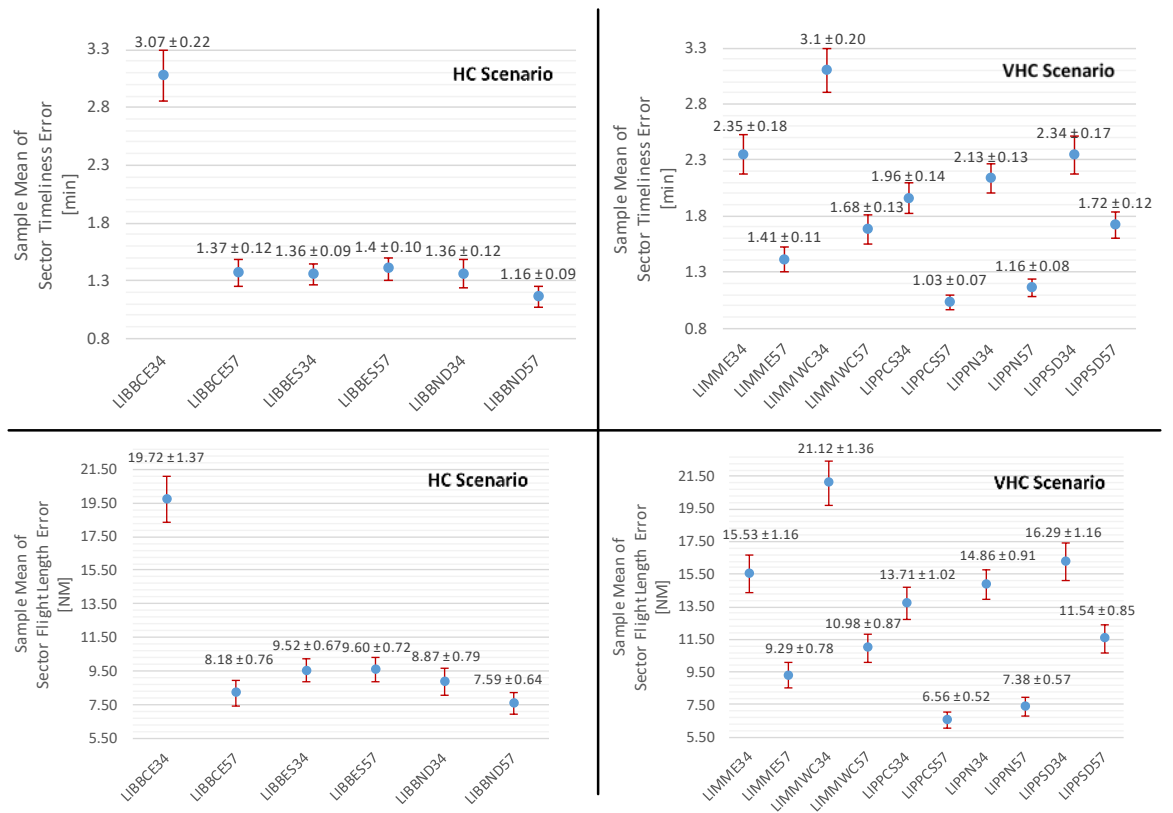

\section{Experimentation for Optimal Sector Collapsing/Decollapsing Configuration}

The support to design has been experimented by means of the following case: the automated and optimal tuning of the configuration of elementary sectors 
in the collapsed sectors in order to optimize controller workload. Thus, the goal of this experimentation case is to support the design of the sector collapsing/decollapsing configuration for a given planned traffic in a performancebased setting. In detail, the performance-oriented objectives are to limit the controller workload from different point of views: the number of communications of EC to FC, the number of EC separation actions, and the number of PC separation actions.

The stated design problem has been faced by formulating an equivalent optimization problem, whose goal is to find an optimal sector collapsing/decollapsing configuration in terms of allocation of elementary sectors in the collapsed sectors, i.e., allocation of PCs and ECs. Clearly, the optimality has been intended with respect to the minimization of specific metrics related to the performance-oriented objectives. Instead, the compositions of collapsed sectors have represented the parameters to be optimally tuned for the solution design. For the experimentation, the traffic scenario has been represented by the planned Italian FRA traffic on 03/07/2019 in the time slot from 12 AM to 2 PM, with only the VHC traffic. However, here the configuration of the scenario is not fixed, but it represents a decision vector variable of the design problem, i.e., it is changed by the optimization module.

In detail, the reference performance metrics have been:

- $\quad$ The total number of EC communication to FC - It is the sum of all the numbers of the EC communication to $\mathrm{FC}$ in the simulated sectors.

- The standard deviation of EC communication to FC - It is the standard deviation of the distribution of the numbers of EC communication to $\mathrm{FC}$ in the simulated sectors.

- The total number of EC separation actions - It is the sum of all the numbers of EC separation actions in the simulated sectors.

- The standard deviation of EC separation actions - It is the standard deviation of the distribution of the numbers of EC separation actions in the simulated sectors.

The total number of PC separation actions - It is the sum of all the numbers of PC separation actions in the simulated sectors.

- $\quad$ The standard deviation of PC separation actions - It is the standard deviation of the distribution of the numbers of PC separation actions in the simulated sectors.

The objectives have been to minimize all the previous metrics. The choice to optimize both the total workload metrics and their standard deviation is significant in order to find the optimal compromise with respect to the possible workload "dimensions". Indeed, on the one hand, the minimization of the total performance metrics penalises the choice of an excessive decollapsing of sectors, i.e., the introduction of a high number of ECs and PCs. On the other hand, the 
minimization of the standard deviations is useful as it aims at rewarding the configurations with a uniform workload for the distributions of ECs and PCs, which will reasonably prevent trajectory-related metrics from negative impacts.

In addition, a safety constraint has been imposed about a maximum threshold for the hourly mean of STCA (Short Term Conflict Alert) conflicts in each collapsed sector. Such constraint has been useful to establish the "border line" between the safe state space and the degraded state space in the search space of solutions. Given that the ABMS engine is already set up with de-conflicting tools (which ensure conflict resolutions and mid-air collision avoidance), it is reasonable to use STCA conflicts as a safety indicator of the traffic complexity. Indeed, if a collapsed sector is subjected to an excessively complex traffic, a degradation will occur in human performance, which may be tracked by measuring STCA conflicts. If such conflicts exceed a critical threshold, the sector is too "wide" for the planned traffic: the current solution is degraded and the AES algorithm will likely decollapse the sector in the next solutions.

We denote with $E S_{a}^{(i)}$ the $i$-th elementary sector in the zone $a$ of the simulated airspace. The index $i$ is associated to the flight level of the elementary sector, thus $i \in\{3,4,5,6,7\}$ for the applied traffic scenarios. Besides, since the proposed experimentation case considers only the VHC traffic, it results that $a \in$ \{LIMME, LIMMWC, LIPPCS, LIPPN, LIPPSD\}. A generic collapsed sector $C S_{a}$ in the zone $a$ is defined as

$$
\begin{gathered}
C S_{a}=E S_{a}^{(i)} \cup E S_{a}^{(j)} \cup \cdots \cup E S_{a}^{(n)}, \\
i, j, \ldots, n \in\{3,4,5,6,7\}, \quad i \neq j \neq \cdots \neq n,
\end{gathered}
$$

$a \in\{$ LIMME, LIMMWC, LIPPCS, LIPPN, LIPPSD $\}$.

Thus, a collapsed sector is the union of different elementary sectors belonging to the same zone $a$. Each EC and each PC is allocated to a single collapsed sector. We also assume that collapsed sectors may be built only by respecting a continuity constraint, i.e., a collapsed sector may be only the union of adjacent elementary sectors with respect to the flight level in the same zone $a$. Thus, notations $a_{j}$ and $a_{j k}$ may be used, i.e.

$$
\begin{gathered}
C S_{a}^{(i)}=a_{j} \stackrel{\operatorname{def}}{\leftrightarrow} C S_{a}^{(i)}=E S_{a_{j},} \\
C S_{a}^{(i)}=a_{j k} \stackrel{\operatorname{def}^{\leftrightarrow}}{ } C S_{a}^{(i)}=E S_{a_{j}} \cup E S_{a_{j+1}} \cup \cdots \cup E S_{a_{k},} \\
j \in\{3,4,5,6,7\}, j<k .
\end{gathered}
$$

For example, LIMME36 $=\{$ LIMME3 $\cup$ LIMME4 $\cup$ LIMME5 $\cup$ LIMME6 $\}$. We denote with $\mathbb{S}_{a}$ the simulated configuration for zone $a$, which is defined as the exhaustive union of non-overlapping collapsed sectors in $a$, i.e. 


$$
\begin{gathered}
\mathbb{S}_{a}=\bigcup_{i=1}^{m} C S_{a}^{(i)}, \quad m \in \mathbb{N}^{*}, \\
C S_{a}^{(j)} \cap C S_{a}^{(k)}=\emptyset, \quad \forall j, k \in\{1, \ldots, m\}, \quad j \neq k, \\
E S_{a_{l}} \subseteq \mathbb{S}_{a}, \quad \forall l \in\{3,4,5,6,7\} .
\end{gathered}
$$

For example, the simulated configuration of LIMME zone is defined as

$$
\begin{gathered}
\mathbb{S}_{\text {LIMME }}=\bigcup_{i=1}^{m} C S_{\text {LIMME }}^{(i)}, \quad m \in \mathbb{N}^{*}, \\
C S_{\text {LIMME }}^{(j)} \cap C S_{\text {LIMME }}^{(k)}=\emptyset, \quad \forall j, k \in\{1, \ldots, m\}, \quad j \neq k,
\end{gathered}
$$

LIMME3 $\subseteq \mathbb{S}_{\text {LIMME }}, \ldots$, LIMME7 $\subseteq \mathbb{S}_{\text {LIMME }}$.

The simulated configuration $\mathbb{S}$ is defined as the tuple of the selected configurations for all the zones $a$ belonging to the reference airspace for the simulation. Thus, for the proposed experimentation case, it results that

$$
\mathbb{S}=\left\langle\mathbb{S}_{\text {LIMME }}, \mathbb{S}_{\text {LIMMWC }}, \mathbb{S}_{\text {LIPPCS }}, \mathbb{S}_{\text {LIPPN }}, \mathbb{S}_{\text {LIPPSD }}\right\rangle
$$

Then, we denote with:

- $\quad \operatorname{Comm}_{\mathrm{EC} \rightarrow \mathrm{FC}}(\mathbb{S})$ the distribution of the cumulated numbers of the EC communication to FC, separation actions,

$S e p_{\mathrm{EC}}(\mathbb{S})$ the distribution of the cumulated numbers of EC separation actions,

in the simulated sectors $C S_{a}^{(i)} \in \mathbb{S}$ at the end of the simulation. We respectively denote with $\sum(\cdot)$ and $\sigma(\cdot)$ the sum (i.e., the total values in $\mathbb{S}$ ) and the standard deviations of the previous distributions. Instead, we denote with $\operatorname{Conf}_{\text {STCA }}\left(C S_{a}^{(i)}, t\right)$ the cumulated numbers of STCA conflicts that have occurred in the simulated sector $C S_{a}^{(i)} \in \mathbb{S}$ until the simulated time $t$. We also denote with $m_{\mathrm{h}}\left(\operatorname{Conf}_{\mathrm{STCA}}\left(C S_{a}^{(i)}\right), t\right)$ the hourly mean of $\operatorname{Conf}_{\mathrm{STCA}}\left(C S_{a}^{(i)}, t\right)$.

We denote with $\mathbf{f}(\mathbb{S})$ the vector objective function of the experimentation case, which contains all the optimization objectives previously mentioned. Thus, it is defined as 


$$
\begin{array}{r}
\mathbf{f}(\mathbb{S})=\left(\sum\left(\operatorname{Sep}_{\mathrm{EC}}(\mathbb{S})\right), \sigma\left(\operatorname{Sep}_{\mathrm{EC}}(\mathbb{S})\right),\right. \\
\sum\left(\operatorname{Sep}_{\mathrm{PC}}(\mathbb{S})\right), \sigma\left(\operatorname{Sep}_{\mathrm{PC}}(\mathbb{S})\right) \\
\left.\sum\left(\operatorname{Comm}_{\mathrm{EC} \rightarrow \mathrm{FC}}(\mathbb{S})\right), \sigma\left(\operatorname{Comm}_{\mathrm{EC} \rightarrow \mathrm{FC}}(\mathbb{S})\right)\right) .
\end{array}
$$

The reference optimization problem for sector collapsing/decollapsing configuration is defined as

$$
\mathbb{S}^{*}=\underset{\mathbb{S}}{\operatorname{argmin}}(\mathbf{f}(\mathbb{S})),
$$

s.t. $\operatorname{Conf}_{\mathrm{STCA}}\left(C S_{a}^{(i)}, t\right)<1, \forall C S_{a}^{(i)} \in \mathbb{S}, \forall t \in \mathbb{R}^{+}$,

with $\mathbb{S}^{*}=\left\langle\mathbb{S}_{\text {LIMME }}, \mathbb{S}_{\text {LIMMWC }}, \mathbb{S}_{\text {LIPPCS }}, \mathbb{S}_{\text {LIPPN }}, \mathbb{S}_{\text {LIPPSD }}\right\rangle^{*}$.

Note that the critical threshold for the hourly mean of STCA conflicts has been set to 1 for each collapsed sector in the solution. A number of 5 iterations has been considered for the convergence threshold of the optimization module. A number of 6 individuals has been applied for the population size at each iteration. Table 2 reports the results of the experimentation case in terms of the convergence history of the evolutionary optimization module. For each solution iteration, both the total metrics (tot.) and the standard deviation metrics (st. dev.) are indicated. Only feasible solutions (i.e., configurations belonging to the safe state space) of a population have been pointed out in the table. Furthermore, a radar chart is reported in Figure 6, showing these design solutions in terms of normalized values for each metric, wherein the normalization is with respect to the maximum value obtained by the solutions. 
Table 2

Convergence History of the AES Module for the Experimentation About Support to Design

\begin{tabular}{|c|c|c|c|c|c|c|c|}
\hline It. & Solution & $\begin{array}{c}\text { EC-FC } \\
\text { Comm. } \\
\text { (tot.) }\end{array}$ & $\begin{array}{c}\text { EC-FC } \\
\text { Comm. } \\
\text { (st. dev.) }\end{array}$ & $\begin{array}{l}\text { EC } \\
\text { Sep. } \\
\text { (tot.) }\end{array}$ & $\begin{array}{c}\text { EC } \\
\text { Sep. } \\
\text { (st. } \\
\text { dev.) }\end{array}$ & $\begin{array}{l}\text { PC } \\
\text { Sep. } \\
\text { (tot.) }\end{array}$ & $\begin{array}{c}\text { PC } \\
\text { Sep. } \\
\text { (st. } \\
\text { dev.) }\end{array}$ \\
\hline 1 & $\begin{array}{l}\text { LIMME37-LIMMWC37-LIPPCS37- } \\
\text { LIPPN37- LIPPSD37 }\end{array}$ & 3332 & 235.65 & 1371 & 81.82 & 3676 & 429.90 \\
\hline 2 & $\begin{array}{l}\text { LIMME3-LIMME4- LIMME5- } \\
\text { LIMME6- LIMME7-LIMMWC3- } \\
\text { LIMMWC4-LIMMWC5-LIMMWC6- } \\
\text { LIMMWC7-LIPPCS3-LIPPCS4- } \\
\text { LIPPCS5-LIPPCS6-LIPPCS7-LIPPN3- } \\
\text { LIPPN4-LIPPN5-LIPPN6-LIPPN7- } \\
\text { LIPPSD3-LIPPSD4- LIPPSD5- } \\
\text { LIPPSD6-LIPPSD7 }\end{array}$ & 5325 & 66.27 & 1836 & 34.59 & 3865 & 55.25 \\
\hline 3 & $\begin{array}{l}\text { LIMME37-LIMMWC37-LIPPCS3- } \\
\text { LIPPCS4-LIPPCS5-LIPPCS6-LIPPCS7- } \\
\text { LIPPN3-LIPPN4-LIPPN5-LIPPN6- } \\
\text { LIPPN7-LIPPSD3-LIPPSD4-LIPPSD5- } \\
\text { LIPPSD6-LIPPSD7 }\end{array}$ & 4195 & 186.94 & 1446 & 82.21 & 3155 & 152.38 \\
\hline 4 & $\begin{array}{l}\text { LIMME34-LIMME57-LIMMWC34- } \\
\text { LIMMWC57-LIPPCS3-LIPPCS4- } \\
\text { LIPPCS5-LIPPCS6-LIPPCS7-LIPPN3- } \\
\text { LIPPN4-LIPPN5-LIPPN6-LIPPN7- } \\
\text { LIPPSD3-LIPPSD4-LIPPSD5-LIPPSD6- } \\
\text { LIPPSD7 }\end{array}$ & 4514 & 130.82 & 1555 & 61.59 & 3330 & 105.18 \\
\hline 5 & $\begin{array}{l}\text { LIMME34-LIMME57-LIMMWC34- } \\
\text { LIMMWC57-LIPPCS34-LIPPCS5- } \\
\text { LIPPCS6-LIPPCS7-LIPPN34- } \\
\text { LIPPN5-LIPPN6-LIPPN7-LIPPSD34- } \\
\text { LIPPSD5-LIPPSD6-LIPPSD7 }\end{array}$ & 4144 & 127.08 & 536 & 33.69 & 3216 & 107.26 \\
\hline
\end{tabular}

The obtained solution (highlighted in Table 2) on average minimizes the objective metrics. Indeed, even if other solutions exhibit better values for some total metrics (e.g., solution 1, which aggregates as much as possible the elementary sectors) or for the standard deviation metrics (e.g., solution 2, which separates as much as possible the elementary sectors), the highlighted solution represents a good compromise between the "extreme" solutions. It qualitatively aggregates the sectors of the ACCs according to their peak of traffic, producing nearly uniform workload distributions for controllers and with a limited total workload. The goodness of the solution is more evident in Figure 6, which shows its good performance with respect to the normalized values of the reference metrics. 
Figure 6

Radar Chart of the Design Solutions and Their Evaluated Performance for the Experimentation Case About Support to Design

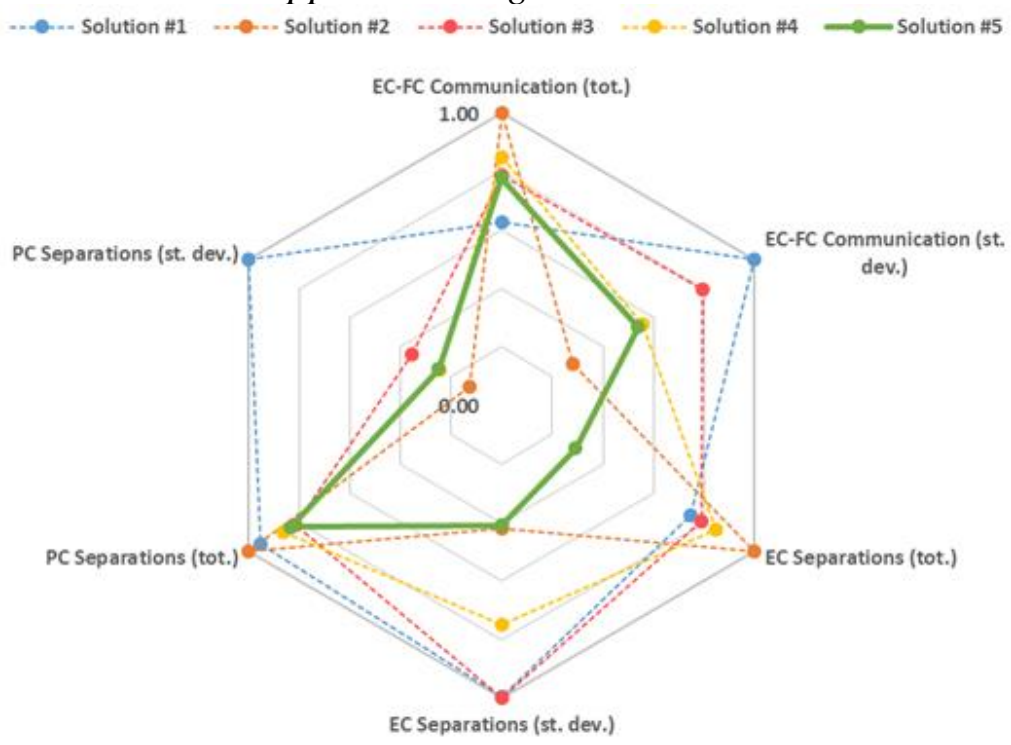

The quality of the obtained solution is further confirmed by Figure 7, which reports the following metrics: the configuration occupancy (i.e., the mean of means of the 10-minutes occupancy over the sectors); the standard deviation of the configuration occupancy (i.e., the standard deviation of means of the 10-minutes occupancy over the sectors); the number of collapsed sectors. It should be noted that such metrics are additional with respect to the experimentation case, in the sense that they are not directly optimized in the problem (7) and they are only influenced by the objective function (6). Anyway, the evaluation of the configuration occupancy provides a "trajectory-related perspective" of the controller workload and of the traffic balancing. Moreover, the number of collapsed sectors is meaningful in order to assess the cost of the proposed configuration in terms of the number of involved ECs and PCs. 


\section{Figure 7}

Occupancy Evaluation and Number of Collapsed Sectors of the Design Solutions for the Experimentation Case About Support to Design

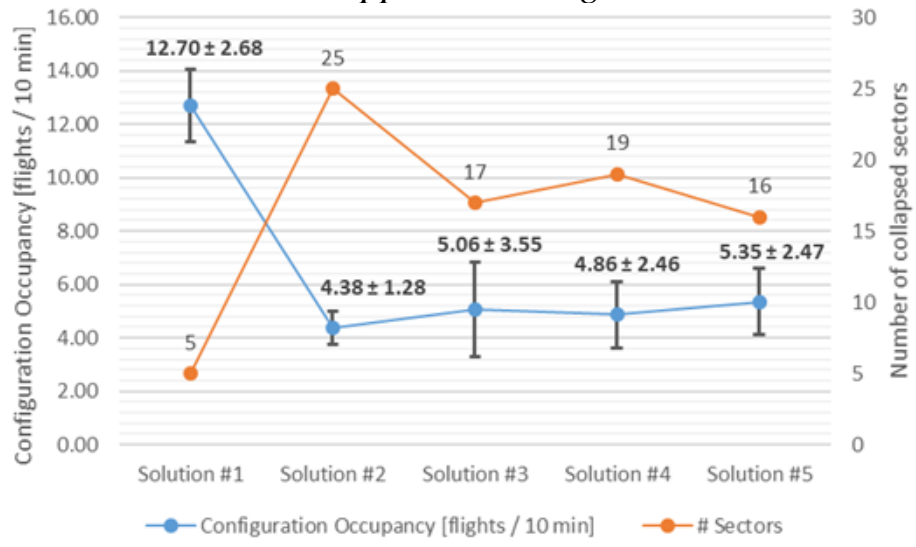

In this respect, solution 1 provides the cheapest configuration, but it exhibits the worst values for the occupancy metrics. To the contrary, solution 2 provides the best values for the occupancy metrics, but it represents the most expensive configuration. Solution 4 also exhibit good values for the occupancy metrics, but it includes three extra collapsed sectors with respect to solution 5. Even if solution 5 has not the best occupancy metrics, its configuration occupancy is similar to those of solutions 3 and 4 and its standard deviation is the same of solution 4 and less than solution 3. Moreover, solution 5 is cheaper with respect to solutions 3 and 4. This confirms the optimality of solution 5, which provides an efficient configuration (from the point of view of controller workload and traffic balancing) with a limited number of controllers.

\section{Conclusion and Future Work}

In this paper, we present the problem of support to design for ATM systems. We propose a methodology for the automated support to identify ATM solutions according to a performance-based setting. The methodology includes the evaluation of the impact on human behaviour and is based on the combination of ABMS and AES paradigms. We prove the soundness of the proposed methodology by carrying out a real case study, which is the transition from DRA to FRA in the Italian airspace. The achieved validation results indicate good agreement between simulated and real flights, with limited deviations for the assessment of performance metrics. Moreover, the optimization of sector collapsing/decollapsing configuration is used to demonstrate the effectiveness of ABMS and AES engines.

Based on our work, we see different fruitful opportunities for future research. For example, the ABMS engine may be enriched with different statistical tools to perform sensitivity analysis, with the injection of "off-nominal" events. 
Besides, the proposed ABMS architecture may be enriched by providing a full automated interpreter for the translation of FRAM-based specifications in agentbased models. 


\section{References}

Bilimoria, K., Sridhar, B., Grabbe, S., Chatterji, G., \& Sheth, K. (2001). FACET: Future ATM Concepts Evaluation Tool. Air Traffic Control Quaterly, 9(1), 1-20.

Blom, H. A., \& Bakker, G. J. (2016). Agent-based modelling and simulation of trajectory based operations under very high traffic demand. Proceedings of the 6th SESAR Innovation Days (SIDs2016). 8-10 November 2016, Delft, The Netherlands: SESAR Joint Undertaking.

Bongiorno, C., Miccichè, S., Ducci, M., \& Gurtner, G. (2015). ELSA air traffic simulator: An empirically grounded agent based model for the SESAR scenario. Proceedings of the 5th SESAR Innovation Days (SIDs2015). 1-3 December 2015, Bologna, Italy: SESAR Joint Undertaking.

Ciruelos, C., Arranz, A., Etxebarria, I., Peces, S., Campanelli, B., Fleurquin, P., . . Ramasco, J. J. (2015). Modelling delay propagation trees for scheduled flights. Proceedings of the 11th USA/EUROPE Air Traffic Management $R \& D$ Seminar (ATM2015). 23-26 June 2015, Lisbon, Portugal: EUROCONTROL.

Deb, K., Pratap, A., Agarwal, S., \& Meyarivan, T. (2002). A fast and elitist multiobjective genetic algorithm: NSGA-II. IEEE Transactions on Evolutionary Computation, 6(2), 182-197. doi:10.1109/4235.996017

Gilbert, N., \& Troitzsch, K. G. (2005). Simulation for the social scientist (2nd ed.). Open University Press.

Herranz, R., Toribio, D., Ramírez, M., Villafáñez, F., Araúzo, J. A., Poza, D., . . Bolic , T. (2015). Price-setting auctions for airport slot allocation: A multiairport case study. Proceedings of the 5th SESAR Innovation Days (SIDs2015). 1-3 December 2015, Bologna, Italy: SESAR Joint Undertaking.

Hollnagel, E. (2012). FRAM: The functional resonance analysis method: Modelling complex socio-technical systems. Ashgate Publishing.

Macal, C. M. (2016). Everything you need to know about agent-based modelling and simulation. Journal of Simulation, 10(2), 144-156. doi:10.1057/jos.2016.7

Molina, M., Martin, J., \& Carrasco, S. (2014). An agent-based approach for the design of the future European air traffic management system. In Y. Demazeau, F. Zambonelli, J. Corchado, \& J. Bajo Pérez (Eds.), Advances in Practical Applications of Heterogeneous Multi-Agent Systems, The PAAMS Collection, PAAM. Lecture Notes in Computer Science, 8473 (pp. 359-362). Springer, Cham. doi:10.1007/978-3-319-07551-8_38

Palumbo, R., Errico, A., Pascarella, D., Gargiulo, F., \& Filippone, E. (2015). Modeling approach for resilience engineering of the future ATM system. Proceedings of the 15th AIAA Aviation Technology, Integration, and 
Operations Conference. 22-26 June 2015, Dallas, Texas, USA: AIAA. doi:10.2514/6.2015-2893

Pasquini, A., Ragosta, M., Silvagni, S., Sujan, M., Rigaud, E., \& Hollnagel, E. (2013). Modelling of automation degradation: A Case study. Proceedings of 3rd SESAR Innovation Days (SIDs2013). 26-28 November 2013, Stockholm, Sweden: SESAR Joint Undertaking.

Pellegrini, A., Di Sanzo, P., Bevilacqua, B., Duca, G., Pascarella, D., Palumbo, R., .. . Gigante, G. (2020). Simulation-based evolutionary optimization of air traffic management. IEEE Access, 8, 161551-161570. doi:10.1109/ACCESS.2020.3021192

Sarker, R. A., \& Ray, T. (Eds.). (2010). Agent-based evolutionary search. Adaptation, Learning, and Optimization, 5. Springer-Verlag Berlin Heidelberg. doi:10.1007/978-3-642-13425-8

SESAR Joint Undertaking. (2017). SESAR solutions catalogue (2nd ed.). doi:10.2829/0404

Šišlák, D., Volf, P., Kopřiva, Š., \& Pěchouček, M. (2011). Agentfly: NAS-wide simulation framework integrating algorithms for automated collision avoidance. Proceedings of 2011 Integrated Communications, Navigation, and Surveillance Conference (pp. F7-1-F7-11). IEEE. doi:10.1109/ICNSURV.2011.5935278

Stroeve, S., Bosse, T., Blom, H. A., Sharpanskykh, A., \& Everdij, M. (2013). Agent-based modelling for analysis of resilience in ATM. Proceedings of the 3rd SESAR Innovation Days (SIDs2013). 26-28 November 2013, Stockholm, Sweden: SESAR Joint Undertaking.

Yliniemi, L., Agogino, A. K., \& Tumer, K. (2014). Evolutionary agent-based simulation of the introduction of new technologies in air traffic management. Proceedings of the 2014 Annual Conference on Genetic and Evolutionary Computation (GECCO'14) (pp. 1215-1222). 12-16 July 2014, Vancouver, Canada: Association for Computing Machinery. doi:10.1145/2576768.2598388 